\title{
openheart Predictors and prognostic value of left atrial remodelling after acute myocardial infarction
}

\author{
Kasper Kyhl, ${ }^{1,2}$ Niels Vejlstrup, ${ }^{1}$ Jacob Lønborg, ${ }^{1}$ Marek Treiman, ${ }^{2}$ \\ Kiril Aleksov Ahtarovski, ${ }^{1}$ Steffen Helqvist, ${ }^{1}$ Henning Kelbæk, ${ }^{1}$ Lene Holmvang, ${ }^{1}$ \\ Erik Jørgensen, ${ }^{1}$ Kari Saunamäki, ${ }^{1}$ Helle Søholm, ${ }^{1}$ Mads J Andersen, ${ }^{1}$ \\ Jacob E Møller, ${ }^{1}$ Peter Clemmensen, ${ }^{1}$ Thomas Engstrøm ${ }^{1}$
}

To cite: Kyhl K, Vejlstrup N, Lønborg J, et al. Predictors and prognostic value of left atrial remodelling after acute myocardial infarction. Open Heart 2015;2:e000223. doi:10.1136/openhrt-2014000223

Received 25 November 2014 Revised 17 April 2015 Accepted 6 May 2015

\section{CrossMark}

\footnotetext{
${ }^{1}$ Department of Cardiology, Rigshospitalet, Copenhagen, Denmark

${ }^{2}$ Department of Biomedical Sciences, Copenhagen University, Copenhagen, Denmark
}

Correspondence to Dr Kasper Kyhl; kasperkyhl@gmail.com

\section{ABSTRACT}

Purpose: Left atrial (LA) volume is a strong prognostic predictor in patients following ST-segment elevation myocardial infarction (STEMI). However, the change in LA volume over time (LA remodelling) following STEMI has been scarcely studied. We sought to identify predictors for LA remodelling and to evaluate the prognostic importance of LA remodelling.

Methods: This is a subgroup analysis from a randomised clinical trial that evaluated the cardioprotective effect of exenatide treatment. A total of 160 patients with STEMI underwent a cardiovascular MR (CMR) 2 days after primary angioplasty and a second scan 3 months later. LA remodelling was defined as changes in LA volume or function from baseline to 3 months follow-up. Major adverse cardiac events were registered after a median of 5.2 years.

Results: Adverse LA minimum volume (LAmin) remodelling was correlated to the presence of hypertension, larger infarct size by CMR, higher peak troponin $\mathrm{T}$, larger area at risk and adverse left ventricular (LV) remodelling. LA maximum volume (LAmax) remodelling was correlated to larger infarct size by CMR, higher peak troponin T, larger area at risk, larger LV mass, impaired LV function and adverse LV remodelling. Kaplan-Meier and Log Rank analyses showed that patients in the highest tertiles of LAmin or LAmax remodelling are at higher risk ( 0.030 and $p=0.018)$.

Conclusions: After a myocardial infarction, LA remodelling reflects a parallel ventricular-atrial remodelling. Infarct size is a major determinant of LA remodelling following STEMI and adverse LA remodelling is associated with an unfavourable prognosis.

\section{INTRODUCTION}

Left atrial (LA) volume is a prognostic predictor across a wide spectrum of cardiac and non-cardiac diseases. ${ }^{1-10}$ During diastole, the mitral valve opens and the pressure in the LA and left ventricular (LV) cavities is equalised. Increased LA pressure will cause LA dilation over time (also known as LA remodelling). ${ }^{11-14}$

\section{KEY MESSAGES}

What is already known about this subject?

- Left atrium maximum volume is a well-known predictor in various diseases. Left atrium dilation over time after an acute myocardial infarction is affected by the same mechanisms as left ventricle diastolic function, such as hypertension, renal impairment, diabetes, ischaemia and left ventricle mass. Previous studies have also identified that adverse left atrium maximum volume remodelling following an acute myocardial infarction is related to impaired outcomes. Left atrium remodelling has mainly been studied using two-dimensional or M-mode echocardiography.

What does this study add?

- We provide cardiovascular MR (CMR) imaging measures of left atrial volume and function remodelling after an acute myocardial infarction. We evaluate predictors of left atrial volume and function remodelling. In the present paper, we demonstrate that a larger final infarct size measured by CMR and troponin T results in adverse left atrium volume remodelling. In addition, we find that both adverse left atrium minimum volume and left atrium maximum volume remodelling is associated with an unfavourable prognosis.

How might this impact on clinical practice?

- We have shown that left atrial volume is affected by infarct severity after an acute myocardial infarction, and the larger the left atrium, the worse the outcome. Owing to costs of MRI, it is unlikely that MRI scanning of patients with STEMI will be implemented as part of routine risk stratification of patients. This study adds to our understanding of cardiac pathophysiology and remodelling after an acute myocardial infarction and adds parameters that affect left atrium volume and function.

LA remodelling is basically influenced by the same mechanisms as LV diastolic function. ${ }^{15}$ LV relaxation and filling pressure after an acute myocardial infarction may be related to the formation of scar tissue. Therefore, following an 
acute myocardial infarction, the LA dilates over time. ${ }^{11-14}$ Thus, we hypothesise that a larger infarct size may lead to reduced LV compliance and consequently increased LV and LA pressures and thereby result in LA enlargement over time.

In terms of LA remodelling, previous studies have focused on LA maximum volume (LAmax). We have recently demonstrated that LA minimum volume (LAmin) and LA fractional change (LAfc) measured immediately after an ST-segment elevation myocardial infarction (STEMI) are better correlated to LV function and infarct size than LAmax and may also be stronger prognostic predictors. ${ }^{16}$ This has been confirmed in a non-STEMI population. ${ }^{8}$ However, the remodelling of LAmin volume and LA function following STEMI has not been studied previously. Also, LAmax remodelling has mainly been studied using two-dimensional or M-mode echocardiography. ${ }^{7} 1718$ Owing to the excellent spatial resolution, accuracy and reproducibility of cardiovascular MR (CMR), it is an ideal method for studying remodelling of LA volumes and LA function over time. ${ }^{19-21}$ CMR also allows for accurate assessment of LV infarct size, area at risk and myocardial salvage. ${ }^{22-26}$

Thus, using CMR, we sought to evaluate (1) the predictors for LA volume (LAmin and LAmax) remodelling and LA function (LAfc) remodelling after STEMI, to improve our knowledge of the dynamics of postinfarction cardiac remodelling, (2) the impact of infarct size on LA remodelling; and (3) the prognostic importance of adaptations in LAmin, LAmax and LAfc.

\section{MATERIALS AND METHODS Study population}

The present study included 160 patients with a first STEMI and symptom duration $\leq 12 \mathrm{~h}$ included in a previously published randomised study evaluating the cardioprotective effect of exenatide treatment. ${ }^{27}{ }^{28}$ STEMI was defined as ST-segment elevation in two contiguous ECG leads of $0.1 \mathrm{mV}$ in $\mathrm{V} 4-\mathrm{V} 6$ or limb leads II, III and augmented vector foot $(\mathrm{aVF})$, or $0.2 \mathrm{mV}$ in leads V1-V3. Patients were not considered for enrolment if they presented with cardiogenic shock or were unconscious. Patients with acute stent thrombosis, known renal insufficiency, atrial fibrillation or previous coronary artery bypass graft surgery were also excluded. All patients eligible for primary percutaneous coronary intervention (PCI) were pretreated with aspirin (300 mg orally or $500 \mathrm{mg}$ intravenously), clopidogrel $(600 \mathrm{mg}$ orally) and unfractionated heparin (10.000 U intravenously) administered before the PCI.

On arrival at the catheterisation laboratory, a coronary angiography was performed to identify the culprit lesion, and primary PCI was performed according to international guidelines. Glycoprotein IIb/IIIa receptor antagonists were administered if no contraindications were present. All patients were treated with clopidogrel $75 \mathrm{mg}$ daily for 12 months and aspirin $75 \mathrm{mg}$ daily indefinitely. Cardiac biomarkers (troponin T) were obtained before intervention, immediately after and at 6 and 12 $18 \mathrm{~h}$. All patients were informed verbally and in writing and all gave their written consent before inclusion. The study was performed according to the Helsinki declaration of good clinical practice and The Danish National Committee on Biomedical Research Ethics approved the protocol. Only patients with two full CMR scans (one at baseline CMR 2 days after STEMI and another after 3 months) were included in the present analysis. No patients had moderate or severe mitral regurgitation evaluated using echocardiography.

\section{CMR acquisition and analysis}

CMR was performed twice; the first scan was performed within a median of 2 days after STEMI (IQR 1-3 days) and the follow-up scan within a median of 89 (IQR 80-93) days after index STEMI on a $1.5 \mathrm{~T}$ scanner (Avanto scanner, Siemens, Erlangen, Germany). LV and LA volumes were assessed using a steady-state free precession cine sequence (slice thickness $8 \mathrm{~mm}$, slice gap $0 \mathrm{~mm}$, echo time $1.5 \mathrm{~ms}$, field of view 300-360 mm, phases 25). Multiple slices in the short-axis image plane were obtained covering the entire cardiac fossa. The area at risk was assessed on the first scan as oedema using a T2-weighted short tau inversion recovery sequence (slice thickness $15 \mathrm{~mm}$, field of view 300-360 mm, inversion time $180 \mathrm{~ms}$, repetition time $2 \mathrm{R}-\mathrm{R}$ intervals, time to echo $65 \mathrm{~ms}$, slice gap $0 \mathrm{~mm}$ ). Final infarct size was assessed on the follow-up CMR scan using a delayed enhancement inversion-recovery sequence (slice thickness $8 \mathrm{~mm}$, slice gap $0 \mathrm{~mm}$, echo time $1.4 \mathrm{~ms}$, field of view $300-360 \mathrm{~mm}$, slice gap $0 \mathrm{~mm}$ ). Images were obtained 10 min after administration of diethylenetriamine pentaacetic acid $(0.1 \mathrm{~mL} / \mathrm{kg}$; Gadovist, Bayer Schering, Berlin, Germany).

All LV and LA volumes were calculated by manually tracing the endocardial border in all 25 time frames in each short axis slide. The papillary muscles were considered as part of the LV cavity. ${ }^{27}{ }^{28}$ The LA appendage was considered as part of the LA volume. LAmax and LAmin were defined as the largest and smallest volumes. LAfc was calculated as follows: ((LAmax_LAmin)/ LAmax). ${ }^{8} 16$ The analysis was performed with ARGUS, Siemens. All LV and LA volumes were standardised according to the body surface area. LV and LA remodelling was defined as an absolute volume change from the baseline scan to the follow-up CMR scan (LA follow-up - LA baseline). A decrease in LA volume was defined as reverse LA remodelling (negative values in the results section) and an increase as adverse LA remodelling (positive values in the results section). A single operator performed all CMR analyses, and all CMR analysis was performed blinded to clinical data as well as to results from the other CMR scan. Interobserver variability was assessed in 50 randomly selected patients with a mean percentage error of $3 \pm 10 \%$ for LAmin, $4 \pm 8 \%$ for LAmax and $1 \pm 11 \%$ for LAfc. ${ }^{16} 21$

The final infarct size was measured using Segment v1.8. ${ }^{25}$ Endocardial and epicardial borders were manually 
traced in short-axis images and the LV mass was calculated. The infarct size (defined as the hyper-enhanced myocardium on the delayed enhancement images) was measured using a semi-automated technique, which has been described previously. ${ }^{26}$ On the T2-weighted shortaxis images, the area at risk was defined as the hyperintensive myocardium with a signal intensity $>2$ SDs of the signal intensity in the normal myocardium. ${ }^{24}$ The salvage index $(\%)$ was calculated as follows: (area at risk -infarct size)/area at risk. ${ }^{23}$

\section{Clinical end point}

Major adverse cardiac events (MACE) were defined as all-cause mortality, reinfarction, admission for congestive heart failure (peripheral or pulmonary congestion) and implantation of an implantable cardioverter defibrillator. In Denmark, each person has a unique social security number that can be used to register hospital admittance and whether the person is alive. A reviewer blinded to all clinical data used this social security number to evaluate readmissions during the follow-up. Follow-up was defined at the time of the baseline scan.

\section{Statistics}

All continuous variables are expressed by their mean (SD) or median (IQR). Binomial variables are expressed as numbers (\%). Study population characteristics are compared according to included and excluded patients (table 1) using $\chi^{2}$ and t tests. Continuous CMR variables were included in a linear regression to LAmin and LAmax remodelling (table 2). CMR determined baseline LAmax was compared with echocardiographic determined baseline LAmax using an xy-plot and a linear regression analysis (figure 3). Normal distribution was tested visually on a histogram. Graphing

\begin{tabular}{lll} 
Table 1 Study population & $\begin{array}{l}\text { Included } \\
\text { population } \\
\text { N (\% of total) }\end{array}$ & $\begin{array}{l}\text { Excluded } \\
\text { population } \\
\text { N (\% of total) }\end{array}$ \\
General & $127(79)$ & $112(79)$ \\
\hline Gender, males & $94(60)$ & $64(45)$ \\
Exenatide treatment & $45(29)$ & $56(44)^{*}$ \\
Systemic hypertension & $78(49)$ & $65(51)$ \\
Hyperlipidaemia & $11(7)$ & $14(11)$ \\
Diabetes mellitus & $70(45)$ & $54(44)$ \\
Infarct localisation, & & \\
anterior & $29(18)$ & $29(20)$ \\
Multiple vessel disease & Mean & Mean \\
& $60.6(10.4)$ & $63.9(12.4)^{\star}$ \\
Age, years. (SD) & $5.3(1.9-7.1)$ & $5.6(1.5-8.0)$ \\
Peak TNT, nag/L (IQR) & $0.9(1.2)$ & $1.1(1.3)$ \\
TIMl flow before & & \\
procedure & $135(56)$ & $122(48)^{*}$ \\
Time, contact to balloon & & \\
*p Value<0.05 compared to study population. & \\
TNT, troponin-T. & &
\end{tabular}

Kaplan-Meier survival function estimates were used to assess proportional hazards for categorical covariates.

We assessed the discriminative power of LAmax, LAmin and LAfc remodelling to predict 5 year event rates of MACE by calculating the area under the curve of the receiver-operating-characteristic (ROC) curve. Further, we assessed the optimal cut-off point as a point on the ROC curve closest to the coordinate $(0,1)$, which can be calculated as $\mathrm{d}=\sqrt{(1-\text { sensitivity })^{2}+(1-\text { specificity })^{2}} \cdot{ }^{29}$ All statistical analysis was performed using ' $\mathrm{R}$ ' (V.3.0.3; R Development Core Team 2014, http://www. R-project.org/). A two-sided p value $<0.05$ was considered statistically significant.

\section{RESULTS}

A total of 387 patients were included in the original study. Of these, 58 patients were excluded (due to aborted STEMI, symptoms duration $>12 \mathrm{~h}$, withdrawal of consent, or Coronary Artery Bypass Surgery Graft), 101 were lost to CMR (32 had contraindications, 30 refused, 12 either died, had reinfarction or stent-thrombosis, 18 refused or were incapable of completing MRI, 4 had a temporary pacemaker, 5 with no reason) and 68 were lost to follow-up or did not undergo the second CMR (figure 1). The excluded patients were older, had a higher incidence of hypertension and had shorter delay from first emergency contact to balloon. Table 1 shows the comparison between included and excluded patients.

Among the included patients, the LAmin was $24.5 \mathrm{~mL} /$ $\mathrm{m}^{2}$ (IQR 18.8-29.0) at baseline and $24.8 \mathrm{~mL} / \mathrm{m}^{2}$ (IQR 18.7-28.6) 3 months after STEMI $(\mathrm{p}=0.40)$. The LAmax was $48.3 \mathrm{~mL} / \mathrm{m}^{2}$ (IQR 41.8-54.1) at baseline and $49.8 \mathrm{~mL} / \mathrm{m}^{2}$ (IQR 40.4-57.2) 3 months after STEMI $(p=0.014)$. The LAfc was $50.1 \%$ (IQR 45.4-54.7) at baseline and 50.9\% (IQR 47.3-56.4) 3 months after STEMI $(\mathrm{p}=0.08)$. There was a wide distribution of LA change and individual patients experienced up to 92\% LAmin remodelling, 61\% LAmax remodelling and 57\% LAfc remodelling during the 3 months following their STEMI (LA remodelling distributions are shown in figure 2). Table 2 shows the linear regression analyses of predictors for LAmin, LAmax and LAfc remodelling. The presence of hypertension is associated with adverse LAmin remodelling, but the LV mass index did only impact LAmax remodelling. Interestingly, the linear regression analyses showed significant association between the final infarct size and adverse LAmin and LAmax remodelling. Similar peak troponin $\mathrm{T}$ was associated with LAmin, LAmax and LAfc remodelling, but area at risk was only associated to LAmin and LAmax remodelling. Left ventricular ejection fraction at baseline was related to LAmax remodelling, but had no association with LAmin remodelling. In general, LA volume remodelling was associated with LV remodelling. Treatment with exenatide was not associated with remodelling of LAmin, LAmax or LAfc. We tested 
Table 2 Univariate linear regression

\begin{tabular}{|c|c|c|c|c|c|c|}
\hline \multirow[b]{2}{*}{ General } & \multicolumn{2}{|c|}{$\mathrm{LA}_{\min }$ remodel } & \multicolumn{2}{|c|}{ LA $_{\max }$ remodel } & \multicolumn{2}{|c|}{$\mathrm{LA}_{\mathrm{fc}}$ remodel } \\
\hline & $\bar{\beta}$ & $p$ Value & $\bar{\beta}$ & p Value & $\bar{\beta}$ & p Value \\
\hline Gender, males & 0.19 & 0.81 & 0.05 & 0.55 & 0.02 & 0.72 \\
\hline Exenatide treatment & 0.002 & 0.98 & 0.07 & 0.39 & 0.04 & 0.60 \\
\hline Systemic hypertension & 0.17 & 0.034 & 0.12 & 0.14 & -0.07 & 0.41 \\
\hline Hyperlipidaemia & -0.03 & 0.76 & 0.04 & 0.59 & 0.06 & 0.45 \\
\hline Diabetes mellitus & -0.09 & 0.27 & -0.05 & 0.58 & 0.10 & 0.21 \\
\hline Multiple vessel disease & 0.10 & 0.19 & 0.08 & 0.32 & -0.04 & 0.60 \\
\hline \multicolumn{7}{|l|}{ General } \\
\hline Age, years & 0.10 & 0.22 & -0.03 & 0.72 & -0.18 & 0.028 \\
\hline Peak TNT & 0.24 & 0.002 & 0.20 & 0.011 & -0.18 & 0.025 \\
\hline \multicolumn{7}{|c|}{ CMR parameters at baseline } \\
\hline LVEDV & -0.06 & 0.44 & 0.05 & 0.54 & 0.09 & 0.28 \\
\hline LVESV & 0.02 & 0.83 & 0.16 & 0.044 & 0.13 & 0.12 \\
\hline LVEF & -0.10 & 0.22 & -0.25 & 0.002 & -0.15 & 0.07 \\
\hline $\mathrm{LA}_{\min }$ & -0.06 & 0.43 & -0.001 & 0.99 & 0.09 & 0.26 \\
\hline $\mathrm{LA}_{\max }$ & -0.09 & 0.27 & -0.14 & 0.07 & -0.06 & 0.44 \\
\hline$L A_{\text {fractional change }}$ & -0.08 & 0.32 & -0.30 & $<0.001$ & -0.29 & $<0.001$ \\
\hline Area at risk & 0.17 & 0.033 & 0.20 & 0.012 & -0.01 & 0.94 \\
\hline \multicolumn{7}{|c|}{ CMR parameters at 3 months } \\
\hline LVEF & -0.34 & $<0.001$ & -0.26 & 0.001 & 0.25 & 0.002 \\
\hline LV mass index & 0.09 & 0.25 & 0.22 & 0.005 & 0.09 & 0.29 \\
\hline Final infarct size & 0.21 & 0.007 & 0.16 & 0.038 & -0.14 & 0.08 \\
\hline Salvage index & -0.12 & 0.12 & -0.08 & 0.30 & 0.13 & 0.10 \\
\hline \multicolumn{7}{|c|}{ CMR parameter differences } \\
\hline LVEDV remodel & 0.57 & $<0.001$ & 0.69 & $<0.001$ & -0.75 & 0.36 \\
\hline LVESV remodel & 0.55 & $<0.001$ & 0.40 & $<0.001$ & -0.39 & $<0.001$ \\
\hline $\mathrm{LA}_{\min }$ remodel & & & 0.73 & $<0.001$ & -0.57 & $<0.001$ \\
\hline $\mathrm{LA}_{\max }$ remodel & 0.73 & $<0.001$ & & & 0.06 & 0.47 \\
\hline $\mathrm{LA}_{\mathrm{fc}}$ remodel & -0.57 & $<0.001$ & 0.06 & 0.47 & & \\
\hline
\end{tabular}

CMR, cardiovascular MR; LAfc, left atrium fractional change; LAmax, left atrium maximum volume; LAmin, left atrium minimum volume; LV, left ventricle; LVEDV, left ventricular end diastolic volume; LVEF, left ventricular ejection fraction; LVESV, left ventricular end systolic volume; TNT, troponin-T.

the correlation between CMR and echocardiography determined LA max and found that a linear correlation exists between the two $(\alpha=0.4, p<0.001$, figure 3$)$.

\section{LA remodelling and outcome}

During the follow-up period of a median of 5.2 years (IQR 4.7-5.8), a total of 36 patients (23\%) experienced MACE (3 patients died due to a cardiac cause, 6 patients died of a non-cardiac cause, 15 patients were admitted due to heart failure, 9 patients suffered a reinfarction and 3 patients had an implantable cardioverter defibrillator). Kaplan-Meier curves of MACE rates stratified by tertiles of LAmin, LAmax and LAfc remodelling are displayed in figure 4. Patients in the highest tertile of LAmin remodelling $\left(>1.6 \mathrm{~mL} / \mathrm{m}^{2}\right)$ and LAmax remodelling $\left(>4.6 \mathrm{~mL} / \mathrm{m}^{2}\right)$ were at a higher risk of suffering a subsequent clinical event $(p=0.030$ and $p=0.018$, respectively), while LAfc remodelling was not associated with MACE $(p=0.90)$. Kaplan-Meier survival function estimates, stratified by the limits of agreement for LAmin, LAmax and LAfc, respectively, are displayed in figure 5 . Patients with LAmin and LAmax remodelling above the $95 \%$ limits of agreement were at a higher risk of suffering a subsequent clinical event $(\mathrm{p}=0.010$ and $\mathrm{p}=0.013$, respectively), while LAfc remodelling was not associated with an unfavourable outcome $(p=0.18)$

Receiver-operating characteristic curves are displayed in figure 6. The area under the curve was 0.66 ( $p=0.002)$ for LAmin remodelling, $0.64(p=0.004)$ for LAmax remodelling and $0.57(\mathrm{p}=0.11)$ for LAfc remodelling. The optimal cut-off value for LAmin remodelling to predict a poor outcome defined by MACE was a $0.8 \mathrm{~mL} / \mathrm{m}^{2}$ increase in LAmin volume and for LAmax remodelling the optimal cut-off value was a $0.1 \mathrm{~mL} / \mathrm{m}^{2}$ increase in LAmax volume.

\section{DISCUSSION}

In this study, we evaluate predictors for LAmin, LAmax and LAfc remodelling following STEMI and assess the impact of LA remodelling on long-term outcome. The main findings of this paper are that adverse LAmin and LAmax remodelling following STEMI are associated with poorer outcome and LA remodelling is determined by final infarct size, peak troponin $\mathrm{T}$, area at risk, the presence of hypertension, LV mass and LV remodelling. The results are considered to increase our knowledge on pathophysiology in a post-STEMI population. 
578 patients admitted with STEMI

191 patients were not included in the original study (Lønborg et al. 20I2)

387 patients randomized to exenatide or placebo

58 were excluded from original study

Due to aborted STEMI (52)

symptom duration (3)

withdrawal of consent (I)

CABG (I)

no reported reason $(I)$

I0I patients were lost to CMR

68 did not have LA data from both CMR.

160 patients included in the present study

Figure 1 Flow chart of study population. CMR, cardiovascular MR; LA, left atrium; ST-segment elevation myocardial infarction (STEMI), ST elevation myocardial infarction; CABG, coronary artery bypass graft surgery.

As mentioned, owing to equalisation of LA and LV pressures during diastole and opening of the mitral valve, LA dilation over time after an acute myocardial infarction is affected by the same mechanisms as LV diastolic function, such as hypertension, renal impairment, diabetes, ischaemia and LV mass. ${ }^{15}$ In this paper, we demonstrate that a larger final infarct size measured by CMR and troponin $\mathrm{T}$ results in adverse LA remodelling, probably through reduced LV compliance and consequently increased LA pressures. Thus, infarct size should be added to the list of predictors for LA remodelling following an acute myocardial infarction.

LAmax increased slightly over time in this study, which is in accordance with previous observations. ${ }^{7}{ }^{30}$ This is the first study to evaluate LAmin remodelling following an acute myocardial infarction. LAmin did not change over time in the general study population. However, there are important differences between LAmin and LAmax that may explain this discrepancy. In STEMI patients, baseline LAmax volume is not associated with acute LV function but determined by pre-existing

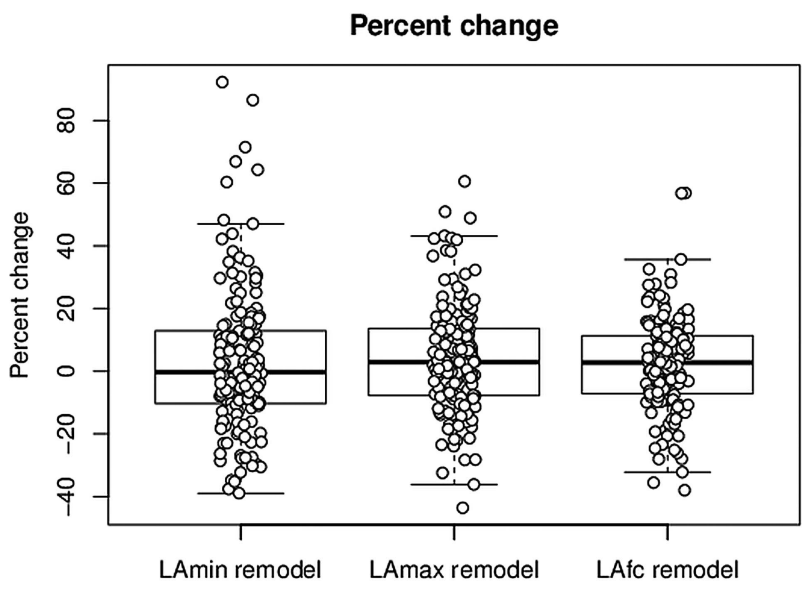

Figure 2 Distribution of left atrial (LA) remodel. Plot showing distribution of left atrium minimum volume (LAmin), LAmax and left atrium fractional change (LAfc) remodelling. Please notice that individual patients experience up to $92 \%$ LAmin remodelling, 61\% LAmax remodelling and 57\% LAfC remodelling during the 3 months following their ST-segment elevation myocardial infarction (STEMI).
Echocardiography vs. cardiac magnetic resonance imaging

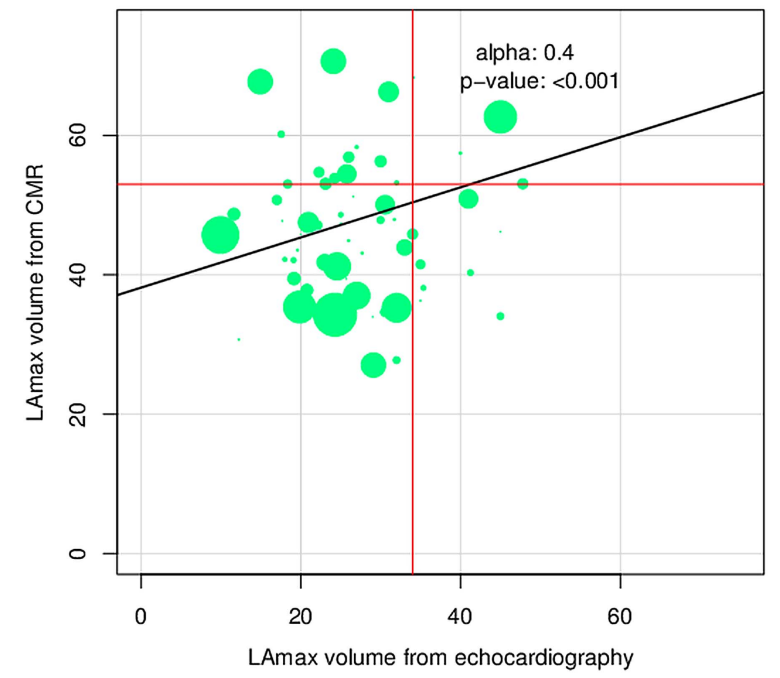

Figure 3 Left atrium maximum volume (LAmax) for echocardiography and cardiovascular magnetic resonance (CMR). Plot comparing echocardiography with cardiovascular MR (CMR) for measurements of left atrial (LA) max volume at baseline. The red lines indicate cut-off values for enlarged LAmax. There is a linear relation between echocardiography and CMR, but please notice that $\alpha<1$. The size of each point is determined by the degree of remodelling during follow-up.

conditions, whereas LAmin volume at baseline is determined by acute changes in LV function, for example, acute stunning and infarct size as well as pre-existing conditions. ${ }^{16}$ LAmax volume is mainly related to longstanding increased LV filling pressure, and LAmax remodelling is attributed to increased filling pressure. LAmin volume is more sensitive towards sudden changes in LV filling than LAmax volume. Both increased LV end-diastolic filling pressure and impeded emptying of the LA due to the reduced longitudinal LV fibre shortening affect LAmin remodelling. This may be an important player immediately after STEMI and probably leads to instant changes in LAmin volume. It may therefore be speculated that change in LAmax after STEMI is a slow adaptation to changing LV filling pressure, whereas change in LAmin over time is affected in the very early phase following STEMI by reduced LV longitudinal fibre contraction and also by changing LV filling pressure over time. Even though we did only observe a small change in mean LAmax and no change in mean LAmin, there were large individual differences in remodelling, far exceeding the $95 \%$ limits of agreement ( figure 2).

Previous studies have identified LV mass, known hypertension, baseline LAmax and baseline estimated glomerular filtration rate as predictors for adverse LAmax remodelling. ${ }^{70}$ This study confirms most of these findings, but also extends the predictors to include LV remodelling, area at risk and extent of myocardial damage and infarct size (peak troponin $\mathrm{T}$ and final infarct size by CMR). The previous studies measure LA volume using 
LA minimum volume remodeling

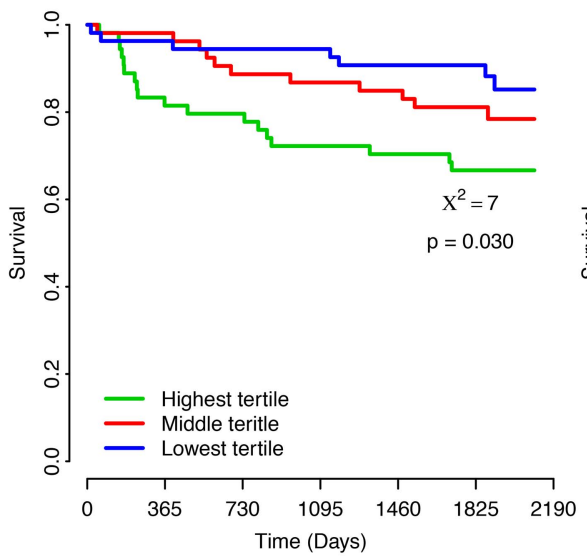

LA maximum volume remodeling

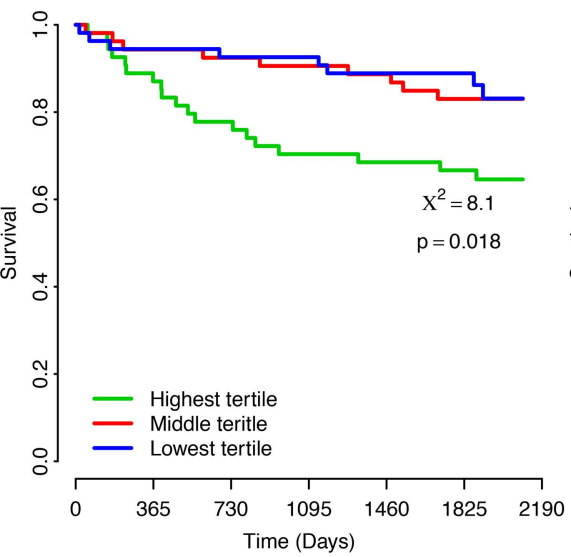

LA fractional change remodeling

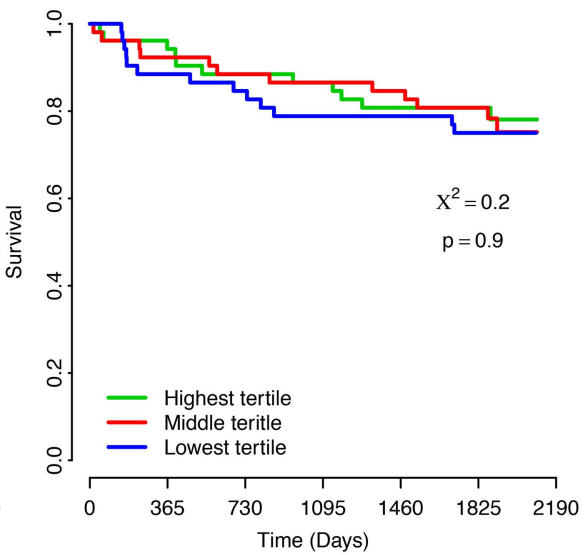

Figure 4 Outcome according to left atrial (LA) remodelling. Outcome stratified by tertile of left atrium minimum volume (LAmin) remodelling, LAmax remodelling and left atrium fractional change (LAfC) remodelling. The curves illustrate the event-free survival from the composite end point.

echocardiography, which is easier to obtain than CMR, but also underestimates the LA volume by $20-35 \% .{ }^{831}$ In our study, there was a good correlation between echocardiography and CMR assessed LAmax at baseline, but echocardiography showed a tendency to underestimate LAmax. Further, it is seen from figure 3 that echocardiography misclassifies a significant amount of patients.

As mentioned, LAmax is a well-known predictor in various diseases. ${ }^{1-10}$ Previous studies have also identified that adverse LAmax remodelling following an acute myocardial infarction is also related to impaired outcomes. ${ }^{7} 17$ Thus, the findings in this study confirm these previous observations. In addition, this study demonstrates that adverse LAmin remodelling is related to poorer outcome. Finally, despite the fact that LAfc remodelling was not related to the outcome in this study, LAfc measured at baseline has been shown to be a strong prognostic predictor. ${ }^{16}$ This study increases our knowledge on the basic pathophysiology of LA remodelling following
STEMI. It also suggests that when studying LA physiology, it is important to look beyond LAmax and assess LAmin and LA function.

\section{Limitations}

A substantial number of potentially eligible patients were not included in this study, which may introduce a selection bias. The excluded patients may represent some of the most critically ill patients. Importantly, there was no difference in peak troponin $T$ between the included and excluded patients. The patients in this study were randomised to either placebo or exenatide, but exenatide treatment did not influence LA remodelling. The sample size is relatively small; thus, the survival analyses presented in this study should be read cautiously due to the limited number of events. Owing to the many statistical comparisons, some significant $p$ values may be observed by chance, and the results must be interpreted with this in mind.
A

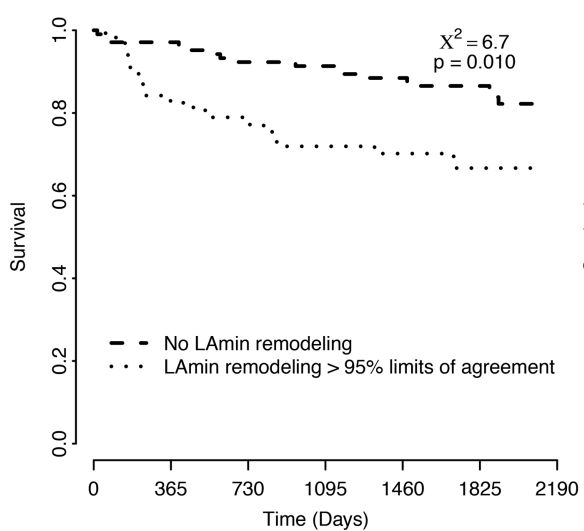

B

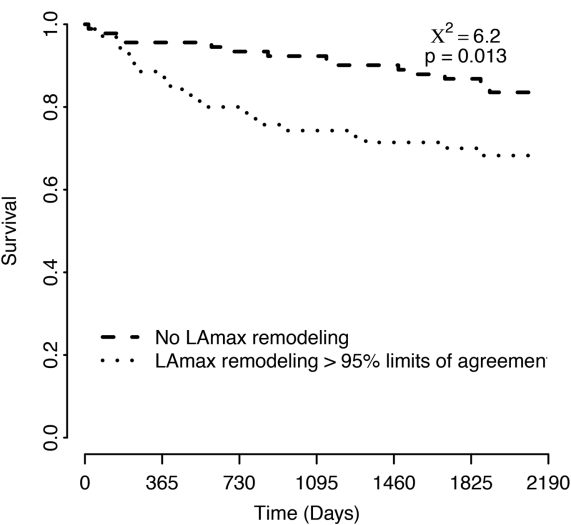

C LA fractional change remodeling

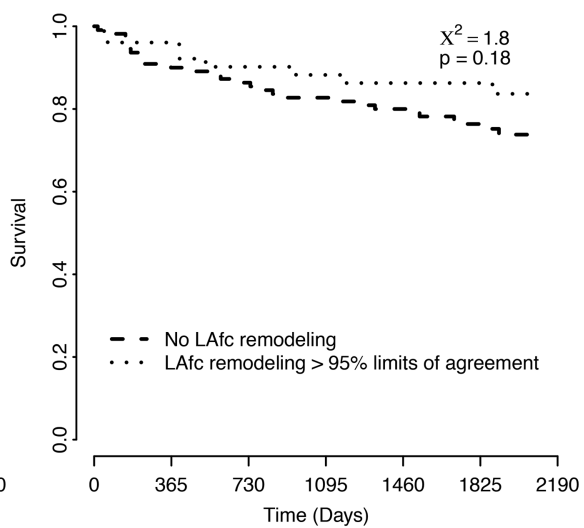

Figure 5 Outcome according to left atrial (LA) remodelling. Outcome stratified by the limits of agreement for left atrium minimum volume (LAmin), left atrium maximum volume (LAmax) and left atrium fractional change (LAfC), respectively. The curves illustrate the event-free survival from the composite end point. 


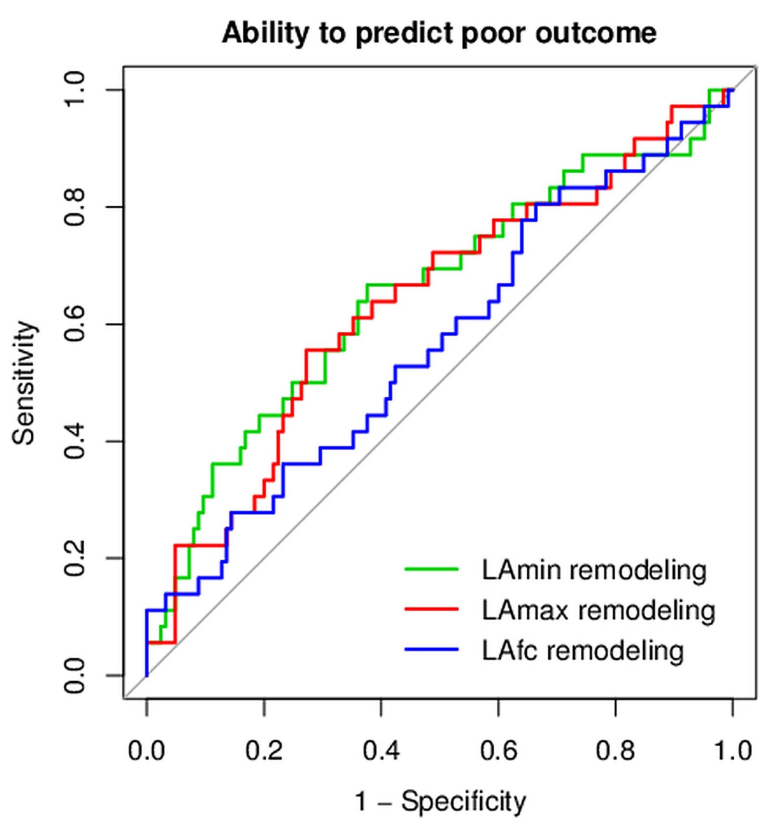

Figure 6 Receiver operating characteristic curve.

Receiver-operating characteristic curves for left atrium minimum volume (LAmin) remodelling, left atrium maximum volume (LAmax) remodelling and left atrium fractional change (LAfC) remodelling displaying the ability to predict major adverse cardiac events after 3 years of follow-up.

\section{Conclusions}

This study extends our pathophysiological understanding of the predictors of LA remodelling to include LV remodelling, area at risk and infarct size. The extent of myocardial damage is a major determinant of LA remodelling following STEMI, and adverse LA remodelling is associated with an unfavourable prognosis and is a poor omen following STEMI.

Acknowledgements The Research Foundation of The Department of Cardiology, Rigshospitalet and The Danish Heart Foundation supported this study financially.

Competing interests None declared.

Ethics approval The Danish National Committee on Biomedical Research Ethics.

Provenance and peer review Not commissioned; externally peer reviewed.

Data sharing statement No additional data are available.

Open Access This is an Open Access article distributed in accordance with the Creative Commons Attribution Non Commercial (CC BY-NC 4.0) license, which permits others to distribute, remix, adapt, build upon this work noncommercially, and license their derivative works on different terms, provided the original work is properly cited and the use is non-commercial. See: http:// creativecommons.org/licenses/by-nc/4.0/

\section{REFERENCES}

1. Genis $A B$, Vazquez R, Puig T. Left atrial enlargement and NT-proBNP as predictors of sudden cardiac death in patients with heart failure. Eur J Heart Fail 2007;9:802-7.

2. Beinart R, Boyko V, Schwammenthal $\mathrm{E}$, et al. Long-term prognostic significance of left atrial volume in acute myocardial infarction. $J A m$ Coll Cardiol 2004;44:327-34. http://linkinghub.elsevier.com/retrieve/ pii/S073510970400796X http://dx.doi.org/10.1016/j.jacc.2004.03.062
3. Benjamin EJ, D'Agostino RB, Belanger AJ, et al. Left atrial size and the risk of stroke and death. The Framingham Heart Study. Circulation 1995;92:835-41. http://eutils.ncbi.nlm.nih.gov/entrez/ eutils/elink.fcgi?

dbfrom=pubmed\&id=7641364\&retmode=ref\&cmd=prlinks http://dx. doi.org/10.1161/01.CIR.92.4.835

4. Cabin HS, Clubb KS, Hall C, et al. Risk for systemic embolization of atrial fibrillation without mitral stenosis. Am J Cardiol 1990;65:1112-16. http://eutils.ncbi.nlm.nih.gov/entrez/eutils/elink. fcgi?dbfrom=pubmed\&id=2330896\& retmode=ref\&cmd=prlinks http:// dx.doi.org/10.1016/0002-9149(90)90323-S

5. Giannuzzi P, Temporelli PL, Bosimini E, et al. Independent and incremental prognostic value of Doppler-derived mitral deceleration time of early filling in both symptomatic and asymptomatic patients with left ventricular dysfunction. J Am Coll Cardiol 1996;28:383-90. http://eutils.ncbi.nlm.nih.gov/entrez/eutils/elink.fcgi? dbfrom=pubmed\&id=8800114\&retmode $=$ ref\&cmd=prlinks

6. Kizer JR, Bella JN, Palmieri V, et al. Left atrial diameter as an independent predictor of first clinical cardiovascular events in middle-aged and elderly adults: the Strong Heart Study (SHS). Am Heart J 2006;151:412-18. http://www.sciencedirect.com/science/ article/pii/S0002870305004631 doi:10.1016/j.ahj.2005.04.031

7. Meris $\mathrm{A}$, Amigoni $\mathrm{M}, \mathrm{Uno} \mathrm{H}$, et al. Left atrial remodelling in patients with myocardial infarction complicated by heart failure, left ventricular dysfunction, or both: the VALIANT Echo Study. Eur Heart $J$ 2008;30:56-65. http://eurheartj.oxfordjournals.org/cgi/doi/10.1093/ eurhearti/ehn499 http://dx.doi.org/10.1093/eurheartj/ehn499

8. Kuhl JT, Møller JE, Kristensen TS, et al. Left atrial function and mortality in patients with NSTEMI. JACC Cardiovasc Imaging 2011;4:1080-7.

9. Reed D, Abbott RD, Smucker ML, et al. Prediction of outcome after mitral valve replacement in patients with symptomatic chronic mitral regurgitation. The importance of left atrial size. Circulation 1991;84:23-34. http://eutils.ncbi.nlm.nih.gov/entrez/eutils/elink.fcgi? dbfrom $=$ pubmed\&id $=2060099 \&$ retmode $=$ ref $\& \mathrm{cmd}=$ prlinks

10. Poulsen MK, Dahl JS, Henriksen JE. Left atrial volume index: relation to long-term clinical outcome in type 2 diabetes. J Am Coll Cardiol 2013;62:2416-21. http://content.onlinejacc.org/article.aspx? articleid=1742655 http://dx.doi.org/10.1016/j.jacc.2013.08.1622

11. Posina K, McLaughlin J, Rhee P, et al. Relationship of phasic left atrial volume and emptying function to left ventricular filling pressure: a cardiovascular magnetic resonance study. J Cardiovasc Magn Reson 2013;15:99.

12. Abhayaratna WP, Seward JB, Appleton CP, et al. Left Atrial Size. J Am Coll Cardiol 2006;47:2357-63. http://linkinghub.elsevier.com/ retrieve/pii/S0735109706008606 http://dx.doi.org/10.1016/j.jacc. 2006.02.048

13. Simek CL, Feldman MD, Haber HL, et al. Relationship between left ventricular wall thickness and left atrial size: comparison with other measures of diastolic function. $J$ Am Soc Echocardiogr 1995;8:37-47. http://eutils.ncbi.nlm.nih.gov/entrez/eutils/elink.fcgi? $\mathrm{dbfrom}=$ pubmed\&id=7710749\&retmode=ref\&cmd=prlinks http://dx. doi.org/10.1016/S0894-7317(05)80356-6

14. Tsang TSM, Abhayaratna WP, Barnes ME, et al. Prediction of cardiovascular outcomes with left atrial size. J Am Coll Cardiol 2006;47:1018-23. http://linkinghub.elsevier.com/retrieve/pii/ S0735109705029219 http://dx.doi.org/10.1016/j.jacc.2005.08.077

15. Thune JJ, Solomon SD. Left ventricular diastolic function following myocardial infarction. Curr Heart Fail Rep 2006;3:170-4. http://eutils. ncbi.nlm.nih.gov/entrez/eutils/elink.fcgi?

dbfrom=pubmed\&id=17129510\&retmode=ref\&cmd=prlinks http://dx. doi.org/10.1007/s11897-006-0018-6

16. Lønborg J, Engstrøm T, Møller JE, et al. Left atrial volume and function in patients following ST elevation myocardial infarction and the association with clinical outcome: a cardiovascular magnetic resonance study. Eur Hear J Cardiovasc Imaging 2013;14:118-27. http://ehjcimaging.oxfordjournals.org/cgi/doi/10.1093/ehjci/jes118 http://dx.doi.org/10.1093/ehjci/jes118

17. Cho JH, Kim SH, Kim C, et al. Prognostic value of left atrium remodeling after primary percutaneous coronary intervention in patients with ST elevation acute myocardial infarction. $J$ Korean Med Sci 2012;27:236. http://synapse.koreamed.org/DOlx.php?id=10. 3346/jkms.2012.27.3.236 http://dx.doi.org/10.3346/jkms.2012.27.3. 236

18. Popescu BA, Macor F, Antonini-Canterin F, et al. Left atrium remodeling after acute myocardial infarction (results of the GISSI-3 Echo Substudy). Am J Cardiol 2004;93:1156-9. http://www. ajconline.org/article/S0002-9149(04)00136-5/fulltext http://dx.doi. org/10.1016/j.amjcard.2004.01.046

19. Järvinen V, Kupari M, Hekali $P$, et al. Assessment of left atrial volumes and phasic function using cine magnetic resonance 
imaging in normal subjects. Am J Cardiol 1994;73:1135-8. http:// eutils.ncbi.nlm.nih.gov/entrez/eutils/elink.fcgi? dbfrom=pubmed\&id=8198044\&retmode=ref\&cmd=prlinks http://dx doi.org/10.1016/0002-9149(94)90298-4

20. Hudsmith L, Petersen S, Francis J, et al. Normal human left and right ventricular and left atrial dimensions using steady state free precession magnetic resonance imaging. J Cardiovasc Magn Reson 2005;7:775-82. http://www.informaworld.com/openurl? genre=article\&doi $=10.1080 / 10976640500295516 \&$ magic $=$ crossrefll D404A21C5BB053405B1A640AFFD44AE3 http://dx.doi.org/10. 1080/10976640500295516

21. Ahtarovski KA, Iversen KK, Lønborg J, et al. Left atrial and ventricular function during dobutamine and glycopyrrolate stress in healthy young and elderly as evaluated by cardiac magnetic resonance. Am J Physiol Heart Circ Physiol 2012;303:H1469-73. http://ajpheart.physiology.org/cgi/doi/10.1152/ajpheart.00365.2012 http://dx.doi.org/10.1152/ajpheart.00365.2012

22. Kim RJ, Fieno DS, Parrish TB, et al. Relationship of MRI delayed contrast enhancement to irreversible injury, infarct age, and contractile function. Circulation 1999;100:1992-2002. http://eutils. ncbi.nlm.nih.gov/entrez/eutils/elink.fcgi? dbfrom=pubmed\&id=10556226\&retmode=ref\&cmd=prlinks http://dx. doi.org/10.1161/01.CIR.100.19.1992

23. Lønborg J, Vejlstrup N, Mathiasen AB, et al. Myocardial area at risk and salvage measured by T2-weighted cardiovascular magnetic resonance: reproducibility and comparison of two T2-weighted protocols. J Cardiovasc Magn Reson 2011;13:50. http://www. jcmr-online.com/content/13/1/50 http://dx.doi.org/10.1186/ 1532-429X-13-50

24. Lønborg J, Engstrøm T, Mathiasen AB, et al. Myocardial area at risk after ST-elevation myocardial infarction measured with the late gadolinium enhancement after scar remodeling and T2-weighted cardiac magnetic resonance imaging. Int $J$ Cardiovasc Imaging 2011;28:1455-64. http://www.springerlink.com/index/10.1007/ s10554-011-9952-9 http://dx.doi.org/10.1007/s10554-011-9952-9

25. Heiberg E, Engblom H, Engvall J, et al. Semi-automatic quantification of myocardial infarction from delayed contrast enhanced magnetic resonance imaging. Scand Cardiovasc $J$ 2005;39:267-75.

26. Lønborg J, Vejlstrup N, Kelbæk H, et al. Final infarct size measured by cardiovascular magnetic resonance in patients with ST elevation myocardial infarction predicts long-term clinical outcome: an observational study. Eur Hear $J$ Cardiovasc Imaging 2013;14:387-95. http://eutils.ncbi.nlm.nih.gov/entrez/eutils/elink.fcgi? $\mathrm{dbfrom}=$ pubmed\&id=23178864\& retmode=ref\&cmd=prlinks http://dx. doi.org/10.1093/ehjci/jes271

27. Lønborg J, Vejlstrup N, Kelbæk H, et al. Exenatide reduces reperfusion injury in patients with ST-segment elevation myocardial infarction. Eur Heart J 2012;33:1491-9. http://eurheartj. oxfordjournals.org/cgi/doi/10.1093/eurheartj/ehr309 http://dx.doi.org/ 10.1093/eurheart//ehr309

28. Lønborg J, Kelbæk H, Vejlstrup N, et al. Exenatide reduces final infarct size in patients with ST-segment-elevation myocardial infarction and short-duration of ischemia.

Circ Cardiovasc Interv 2012;5:288-95. http://eutils.ncbi.nlm.nih.gov/ entrez/eutils/elink.fcgi?dbfrom $=$ pubmed\&id $=22496084 \&$ retmode $=$ ref\&cmd=prlinks http://dx.doi.org/10.1161/CIRCINTERVENTIONS. 112.968388

29. Hajian-Tilaki K. Receiver operating characteristic (ROC) curve analysis for medical diagnostic test evaluation. Casp J Intern Med 2013;4:627-35.

30. Antoni ML, ten Brinke EA, Marsan NA, et al. Comprehensive assessment of changes in left atrial volumes and function after ST-segment elevation acute myocardial infarction: role of two-dimensional speckle-tracking strain imaging. J Am Soc Echocardiogr 2011;24:1126-33. http://linkinghub.elsevier.com/ retrieve/pii/S0894731711004755 http://dx.doi.org/10.1016/j.echo. 2011.06.017

31. Rodevan O, Bjornerheim R, Ljosland M, et al. Left atrial volumes assessed by three- and two-dimensional echocardiography compared to MRI estimates. Int J Card Imaging 1999;15:397-410. http://eutils.ncbi.nlm.nih.gov/entrez/eutils/elink.fcgi? dbfrom=pubmed\&id=10595406\& retmode=ref\&cmd=prlinks http://dx. doi.org/10.1023/A:1006276513186 